\title{
A New Method for Tuning PID Parameters by Using Clone Selection Algorithm
}

\author{
Weiyong Huang ${ }^{1, *}$, Yuqing $\mathrm{Gao}^{2}$ and Xiuling Tian² \\ ${ }^{1}$ School of Information and Electrical Engineering, Xuzhou Institute of Technology, Xuzhou Jiangsu 221111- China \\ ${ }^{2}$ Jiangsu Key Laboratory of Large Engineering Equipment Detection and Control, Xuzhou Institute of Technology, Xuzhou Jiangsu \\ 221111- China
}

Received 15 May 2013; Accepted 25 July 2013

\begin{abstract}
In order to improve the control performance of PID controller, a new method based on an improved clone selection algorithm (CSA) and the criteria of monotonic fitness function was introduced. Experimental results on 3 typical control systems (second order control system, high order control system, and second order control system) indicated that the proposed method could obtain optimum PID parameters and have better control performance than those methods based on GA and PSO methods, with faster response speed, shorter adjusting time and smaller or no overshoot.
\end{abstract}

Keywords: PID controller; parameter optimization, fitness function, clone selection algorithm

\section{Introduction}

Although control theory has been developed significantly for linear systems, the PID controllers are still widely used in industrial control processes because of its simple structure and robustness[1]. Because PID controller works based on the combination of error's proportional, integral and derivative and the control performance depends entirely on selection of 3 parameters ( $\mathrm{kP} 、 \mathrm{kI}$ and $\mathrm{kD})$, it is necessary to have effective and efficient approaches to optimize the control parameters automatically[2].

Today some intelligent methods, such as fuzzy theory, genetic algorithm (GA) and particle swarm optimization (PSO), have been proposed to optimize the parameters of PID controllers[3],[4],[5]. However, the above algorithms have their own defects. Fuzzy theory requires more prior knowledge of controlled object, GA can not find the optimum solution, and PSO algorithm easily appear premature convergence and low search precision [6],[7],[8].

Clone selection algorithm (CSA) by Castro, according to the theory of immunity in the clone selection mechanism, is a new optimization algorithm with heuristic intelligence, which not only has a faster convergence speed in the search range, but also can overcome the problem of premature convergence that many evolutionary computation can not overcome [9],[10]. clone selection algorithm is expected to be an alternative potential algorithm to obtain an optimum PID controller with the superior performances over the classical approaches.

In this paper, a new tuning method for PID controller parameters by using an improved clone selection algorithm was introduced and applied to design PID controllers for 3 typical control systems in an attempt to enhance the control

\footnotetext{
*E-mail address: h_weiyong@163.com

ISSN: 1791-2377 @ 2013 Kavala Institute of Technology. All rights reserved.
}

performance. The Experimental results showed that the proposed method is feasible, and can yield much lower errors than GA and PSO method

This paper is organized as follows: Section 1 outlines briefly the problem and related work. Section 2 states the principle of PID control algorithm. Section 3 presents the principle and algorithm about CSA to be used for tuning PID parameters. 3 typical control system simulation experiment and its results using the approach proposed in the paper are presented in Section 4, followed by the conclusion summarized in Section 5.

\section{Principle of PID control algorithm}

The control system consists of the controller and the controlled object, in which the PID controller includes proportional, integral and derivative units. The PID control is implemented by a linear combination of the proportional, differential and integral. The output of controller is used to control the controlled object, as shown in Figure1, where $r(t)$ is the system input and $y(t)$ is the system output.

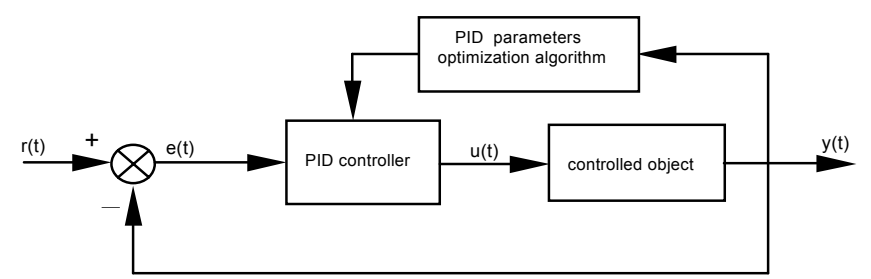

Fig. 1 Block diagram of PID control system principle

The proportional coefficient KP is used to speed up the response of the system and to improve the regulating accuracy of the system. The integral coefficient KI is used to eliminate static error. The differential coefficient $\mathrm{KD}$ reflects 
the varying trend of deviation signal. The output of PID controller can be described as:

$$
\mathrm{u}(\mathrm{t})=\mathrm{k}_{\mathrm{P}}\left[\mathrm{e}(\mathrm{t})+\mathrm{K}_{\mathrm{I}} \int_{0}^{\mathrm{t}} \mathrm{e}(\mathrm{t}) \mathrm{dt}+\mathrm{K}_{\mathrm{D}} \frac{\mathrm{de}(\mathrm{t})}{\mathrm{dt}}\right]
$$

Where $t$ represents the sample number, $u(t)$ indicates the controller output,e(t) represents the deviation of the system input signal.

In PID control system, whether the selection of PID parameters is correct or not has a very important influence on the performance of the control system. PID Parameters selection of KP, KI and KD in formula 1 is an issue of multiobjective optimization. The traditional parameter optimization method is suitable for single-objective linear method, so it is difficult to simultaneously take into account the parameters of performance indicators. At present some intelligent methods have been used to optimize the parameters of PID controllers. In order to overcome defects of ordinary methods and to obtain optimal parameters of PID controller, an improved clone selection algorithm for PID Parameters selection and optimization is proposed in the paper.

\section{Improved Clone Selection Algorithm And PID Parameters Optimization}

The basic principle of clone selection algorithm is that the function to be optimized and its constraints is viewed as antigen, the solution of the problem is viewed as antibody and the affinity between the antibody, antibody-antigen viewed as the objective function of the problem[11]. In order to tune automatically the PID parameters, an improved clone selection algorithm is outlined in the paper. Its block diagram is shown in Figure2. The corresponding steps are explained in detail as follows:

Step 1: Initialization: Randomly generate $\mathrm{n}$ antibodies corresponds to a possible solution, as the initial populations A, constituting the initial population space In.

Step 2: Evaluation and selection: Calculate the affinity function of antibody in the population, and divide $\mathrm{N}$ antibodies into two parts : candidate set $\mathrm{Ar}$ and memory set Am .

Step 3: Cloning: Clone $\mathrm{k}$ antibodies with the highest affinity, cloning number being proportional to the affinity.

Step 4: Mutation: According to a specified probability, randomly perform the operation of mutation on the cloned antibodies at a certain scale.

Step 5: Re-evaluation and re-selection: Re-calculate the affinity of antibodies cloned and mutated. If the affinity is even higher than the original, replace the original antibody with the new antibody, so as to form a new memory set.

Step 6: Extinction: Delete $\mathrm{d}$ antibodies with lowest affinity in Ar, generate randomly $d$ antibodies, and add them to the population, in order to ensure the diversity of antibodies, which simulate 5\% B cells naturally dying process in the biological clone selection.

Step 7: Stop: If the stop criterion is satisfied, then output the result, or else return to step 2.

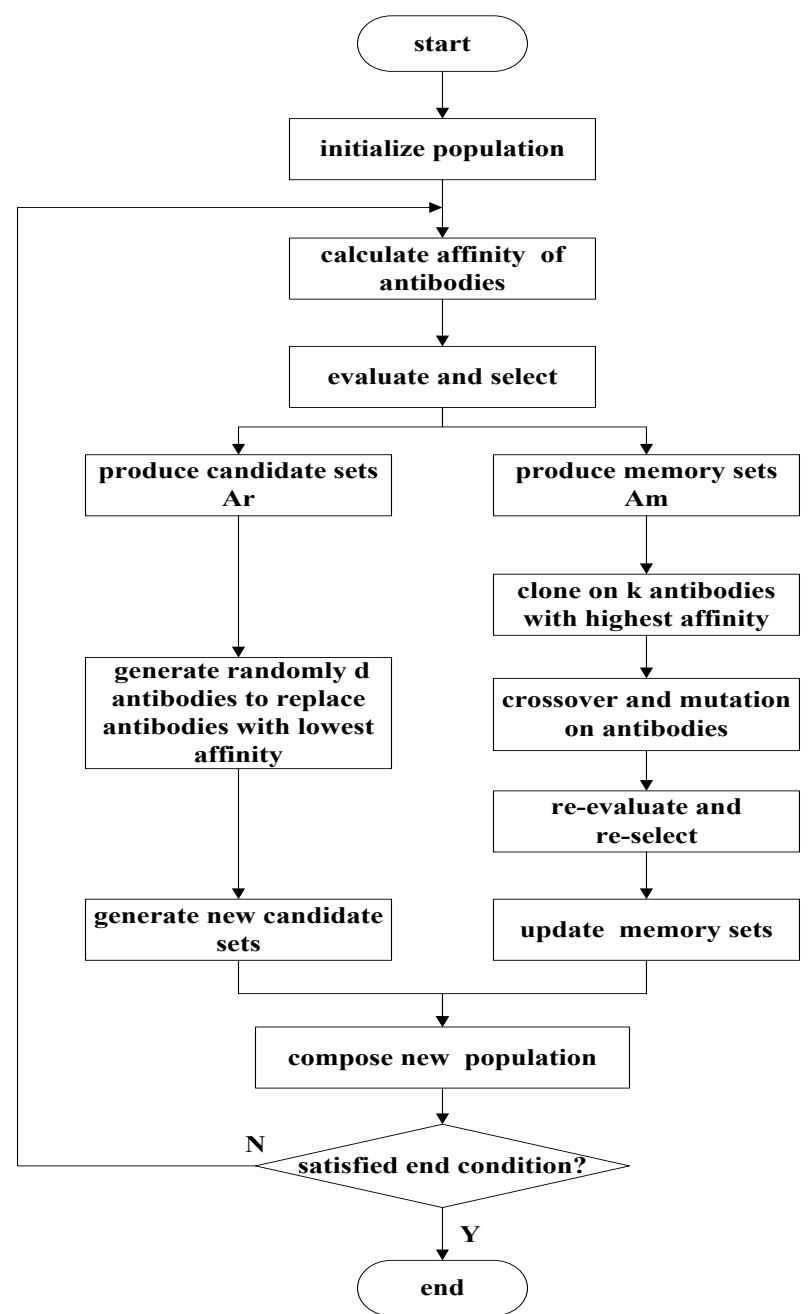

Fig. 2 Block diagram of improved clone selection algorithm

On the basis of improved clone selection algorithm, a new approach for PID parameters optimization was presented in the this paper. The block diagram of PID parameters optimization is illustrated in Figure 3.

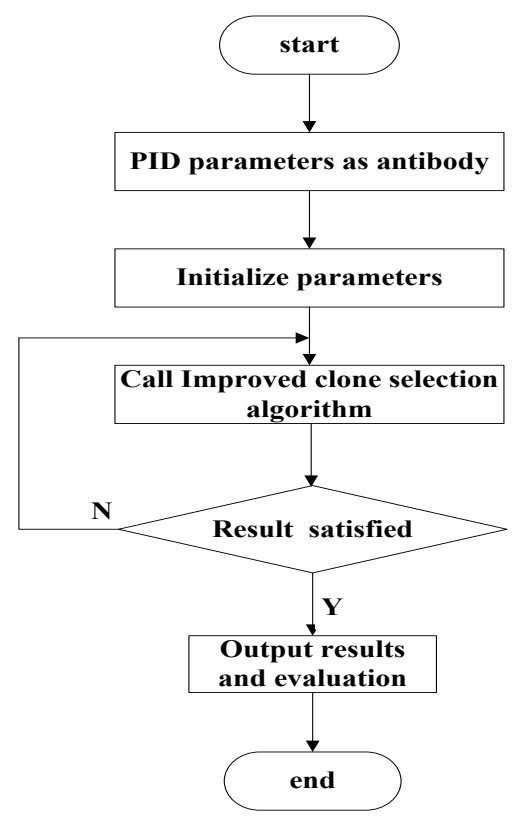

Fig. 3 Block diagram of improved clone selection algorithm 


\section{Experiment and Results}

As the optimization performance is influenced deeply by fitness function, the first step is to design fitness function. Research showed that all conventional monotonic fitness function can not satisfy for some individuals in a population, and non-monotonic fitness function is necessary so as to obtain excellent comprehensive performance indexes. In this paper to ensure that the PID control parameters will produce good step response curve and the smallest time-domain performance indexes, maximum overrun, rising time, adjustable time, steady-state error are adopted as evaluation items. The fitness function can be stated in formula 2 .

$$
f=\frac{1}{\left(1-e^{-\beta}\right)\left(\delta+e_{s s}\right)+e^{-\beta}\left(t_{s}+t_{r}\right)}
$$

Where $\delta, e_{s s}, t_{s}, t_{r}$ stand for maximum overshoot, steady state error, setting time (with $2 \%$ error allowance), and rise time, respectively. $\beta$ is weight coefficient, which is selected on the base of the control system's requirements, such as promptness, exactness and stability. In this paper, $\beta=1$.

The parameters of the CSA used during following particular examples are set, shown in Table 1.

Table 1. Parameters settings of CSA

\begin{tabular}{cc} 
Table 1. Parameters settings of CSA & Setting \\
\hline CSA parameter & 100 \\
Total generation & 100 \\
Initial population size & 22 \\
Length of coding & 10 \\
Clone factor & 5 \\
Suppression threshold & 0.9 \\
Cross probability & 0.01 \\
Mutation probability &
\end{tabular}

To verify the PID parameters optimization approach, 3 different system models were constructed in MATLAB. The algorithm was coded in MATLAB6.5 and the simulation experiments were run on an Intel(R) core(TM) i3-2120CPU (a) $3.3 \mathrm{GHz}$ with $2.00 \mathrm{~GB}$ memory capacity.

In order to cover typical kinds of common industrial processes, the following 3 typical control models had been taken as examples:

Model I: second order control system

$$
\mathrm{G}_{1}(\mathrm{~s})=\frac{1.6}{\mathrm{~s}^{2}+2.584 \mathrm{~s}+1}
$$

The PID parameters, the step response curves, and the performance indexes by 3 kinds of optimization method for Model I are shown in Table2, Figure 4, Table 3, respectively.

Table 2. Obtained parameters by 3 methods for model I

\begin{tabular}{cccc}
\hline method & $\mathrm{K}_{\mathrm{P}}$ & $\mathrm{K}_{\mathrm{I}}$ & $\mathrm{K}_{\mathrm{D}}$ \\
\hline GA & 1.0251 & 0.9386 & 0.0973 \\
PSO & 1.2828 & 0.9626 & 0.1200 \\
CSA & 1.7566 & 1.1986 & 0.3496 \\
\hline
\end{tabular}

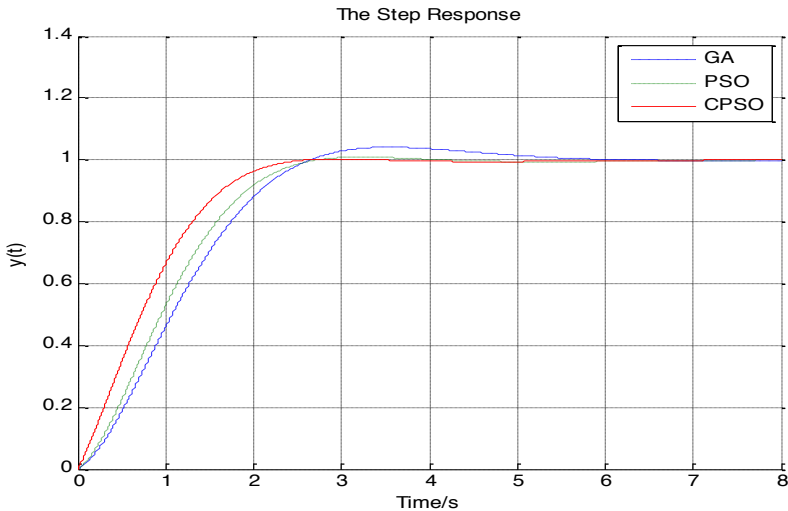

Fig. 4 Step response curves of 3 methods for model I

Table 3. Performance indexes s by 3 methods for model I

\begin{tabular}{cllll}
\hline method & ts & tr & $\delta$ & ess \\
\hline GA & 4.7442 & 2.4906 & 0.0411 & 0 \\
PSO & 2.4347 & 2.4347 & 0.0094 & 0 \\
CSA & 2.1820 & 2.1820 & 0 & 0 \\
\hline
\end{tabular}

Form figure 4 and Table 3, it can be seen that: (1) by using CSA algorithm proposed in this paper, the PID controller has better control performance, obtaining the fastest response, the shortest setting time, minimal dynamic error, and no overshoot or steady state error; (2) PSO algorithm can achieve control of the effect is better than GA, but still exists a little overshoot; (3) the optimization effect of the GA algorithm is the worst, overshoot maximum, and adjust time the longest.

Model : high order control system

$$
G_{2}(s)=\frac{6}{s^{4}+3 s^{2}+4 s^{2}+3 s+1}
$$

The PID parameters, the step response curves, and the performance indexes by 3 kinds of optimization method for Model II are shown in Table4, Figure 5, Table 5, respectively.

Table 4. Obtained parameters by 3 methods for model II

\begin{tabular}{cccc}
\hline method & $\mathrm{K}_{\mathrm{P}}$ & $\mathrm{K}_{\mathrm{I}}$ & $\mathrm{K}_{\mathrm{D}}$ \\
\hline GA & 0.0537 & 0.0251 & 0.0120 \\
PSO & 0.0619 & 0.0352 & 0.0221 \\
CSA & 0.0987 & 0.0490 & 0.1071 \\
\hline
\end{tabular}

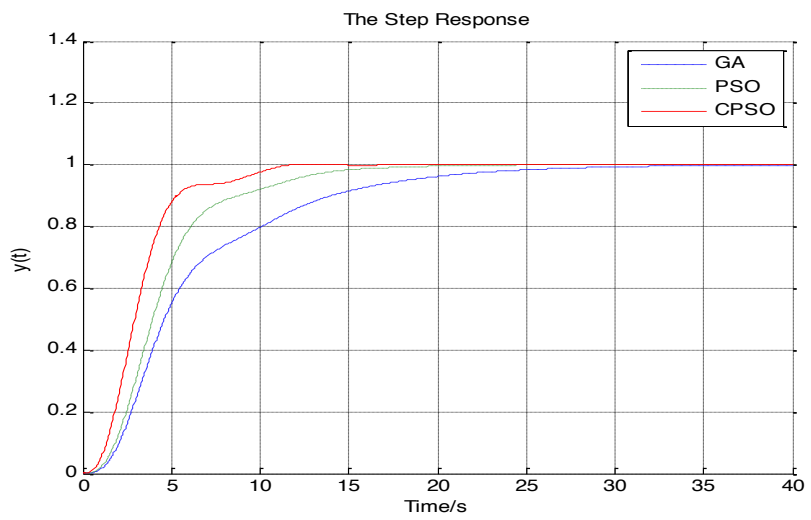

Fig. 5 Step response curves of 3 methods for mode II 
Table 5. Performance indexes s by 3 methods for model II

\begin{tabular}{ccccc}
\hline method & $\mathrm{t}_{\mathrm{s}}$ & $\mathrm{t}_{\mathrm{r}}$ & $\delta$ & $\mathrm{e}_{\mathrm{ss}}$ \\
\hline GA & 23.8181 & 23.8181 & 0 & 0 \\
PSO & 14.3717 & 14.3717 & 0 & 0 \\
CSA & 9.6552 & 9.6552 & 0 & 0 \\
\hline
\end{tabular}

Form figure 5 and Table 5, it was demonstrated that: (1) the control performance of CSA method is better obviously than that of GA and PSO methods, having the fastest response, the shortest setting time, and no overshoot or steady state error. (2) The control performance of PSO method is better than that of GA method.

Model : second order system with pure time-delay

$$
G_{3}(s)=\frac{1}{s^{2}+2 s+1} e^{-0.5 s}
$$

The PID parameters, the step response curves, and the performance indexes by 3 kinds of optimization method for Model III are expressed in Table6, Figure 6, Table 7, respectively.

\begin{tabular}{cccc}
\multicolumn{4}{c}{ Table 6 Obtained parameters by 3 methods for mode III } \\
\hline method & $\mathrm{K}_{\mathrm{P}}$ & $\mathrm{K}_{\mathrm{I}}$ & $\mathrm{K}_{\mathrm{D}}$ \\
\hline GA & 2.5736 & 1.4420 & 1.4977 \\
PSO & 2.1984 & 1.3364 & 1.2548 \\
CSA & 2.0460 & 1.0724 & 1.1831 \\
\hline
\end{tabular}

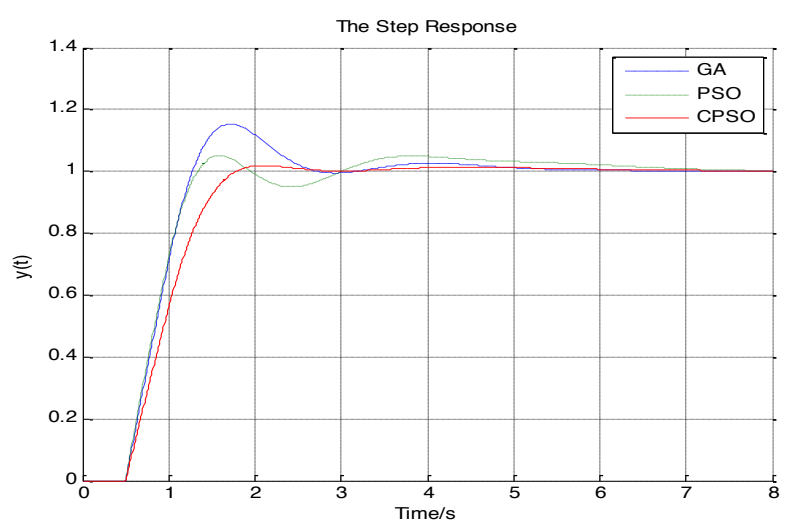

Fig. 6 Step response curves of 3 methods for mode III
Table 7 Performance indexes s by 3 methods for model III

\begin{tabular}{ccccc}
\hline method & $\mathrm{t}_{\mathrm{s}}$ & $\mathrm{t}_{\mathrm{r}}$ & $\delta$ & $\mathrm{e}_{\mathrm{ss}}$ \\
\hline GA & 4.5531 & 1.2496 & 0.1526 & 0 \\
PSO & 4.9770 & 1.4647 & 0.0924 & 0 \\
CSA & 1.6763 & 1.6763 & 0.0191 & 0 \\
\hline
\end{tabular}

According to figure 6 and Table 7, it was illustrated that: (1) although rise time by CSA algorithm is a slightly longer, but setting time and overshoot are minimal, the comprehensive quality of control system by CSA algorithm being clearly better than that of GA and PSO method. (2) GA method has the worst control performance.

\section{Conclusions}

In this paper, a new method for tuning PID parameters was introduced by using clone selection algorithm and the criteria of monotonic fitness function. The Simulation results on 3 typical control systems (second order control system, high order control system, and second order control system) demonstrated that the control systems by using the proposed method to tune PID parameters have better control performance, compared to the conventional GA and PSO method. Future work includes more empirical experimentation with monotonic fitness functions to see different fitness functions have what influence on the performance of control systems.

\section{Acknowledgment}

The authors are grateful to the support of the Open Foundation of Jiangsu Key Laboratory of Large Engineering Equipment Detection and Control (JSKLEDC201212), and the Innovation Team Foundation of Xuzhou Institute of Technology.

\section{References}

1. Wang Wei, Zhang Jingtao, Chai Tianyou,"Asurvey of advanced PID parameter tuning methods", Acta Automatica Sinica, 26(3),2000, pp.347-355.

2. Guo Da-qing,LI Xiao,Zhao Yong-jin, "Self-tuning of PID parameters based on improved PSO algorithm", Computer Engineering, 38(18), 2007, pp.202-204.

3. Lin Jun,Ni Hong,Sun Peng,etc, “.Adaptive resource allocation based on neural network PID control”, Journal of Xi'an Jiaotong university, 47(4), 2013, pp.112-117.

4. Jiang Shan,Feng Wenhao,Yang Zhiyong,etc,"Pneumatic control technology based on fuzzy PID for MRI compatible robots", 34(5), 2012, pp.532-538.

5. Fu Xiaoyun,Fang Xu,Yang Gang,etc,“ Design and simulation ogGA-based PID controller", J. Huazhong Univ. of Sci. \& Tech. (Natural Science Edtion), 40(5), 2012,pp.1-5..

6. Jaafar, H.I.,Mohamed, Z.,Abidin, A.F.Z.,etc,“ PSO-tuned PID controller for a nonlinear gantry crane system", Proceedings - 2012 IEEE International Conference on Control System, Computing and Engineering, Penang, Malaysia, IEEE,2013, pp.515-519.
7. Hu Hai-bing, Hu Qing-bo, LU Zheng-yu, “ PID controller design in servo system based on particles warm optimization", Journal of Zhejiang University (EngineeringScience),40(12),2006,pp.21442148.

8. Bergh, F V D, Engelbrecht, A P, “Cooperative learning in neural networks using particle swarm optimizers”, S. Afr. Comput. J. (26), 2000, pp,84-90.

9. De Castro L N, Von Zuben F J,"Learning and optimization using the clonal selection principle", IEEE Transactions on Evolutionary Computation, 6(3),2002, pp.239-251.

10. Fang Xian-jin,Li Long-shu," Convergence proof for generic clonal selection algorithm", Application Research of Computers, 27(5), 2010, pp.1683-1685.

11. Bi Xiaojun,"Information intelligent processing technology", Beijing,Publishing House of Electronics industry, 2010, pp.313-316. 